- Published: 21 October 2017

\title{
Wear resistance coatings of nickel alloys obtained by supersonic plasma torch
}

- V. I. Kuzmin,

- N. A. Rudenskaya,

- N. V. Sokolova \&

- D. V. Sergachev

Journal of Friction and Wear volume 38, pages395-400 (2017)

\section{Abstract}

The research results of coatings of nickel alloys deposited using supersonic atmospheric-plasma spraying are presented. The main feature of the work carried out is that the plasma flow is charged by powder material using the powder ring injector designed by authors. The ring powder injector unit is equipped by a gas dynamic focusing system that makes it possible to ensure the conditions of creating coatings which are not subject to wear and tear under abrasive friction.

\section{References}

1. 1 .

Borisov, Yu.S., Kharlamov, Yu.A., Sidorenko, S.L., et al., Gazotermicheskie pokrytiya iz poroshkovykh materialov (Gas-

Thermal Coatings form Powder Materials), Kiev: Naukova Dumka, 1987.

\section{Google Scholar}

2. 2 .

Klinskaya-Rudenskaya, N.A., Kopysov, V.A., and Kotsot, S.V., Peculiarities of $\mathrm{Ni}-\mathrm{Cr}-\mathrm{B}-\mathrm{Si}$ alloysbased composite coatings: wear resistance, Fiz. Khim. Obrab. Mater., 1994, no. 6, pp. 52-57. 
3. 3 .

Rudenskaya, N.A., Shveikin, G.P., and Rudenskaya, M.V., Specific features of structuring of the main layer of a metal-ceramic coating, Dokl. Chem., 2011, vol. 441, no. 1, pp. 350-353.

\section{Article Google Scholar}

4. 4 .

Shveikin, G.P., Rudenskaya, N.A., Sokolova, N.V., Kuz'min, V.I., Kartaev, E.V., and Sergachev, D.V., Supersonic plasma and wearresistant nickel alloy coatings, Dokl. Chem., 2015, vol. 463, no. 1, pp. 194-197.

\section{Article Google Scholar}

5. 5 .

Rudenskaya, N.A., Shveikin, G.P., Sokolova, N.V., and Rudenskaya, M.V., Pulse division of plasma spheroids, Dokl. Chem., 2009, vol. 429, no. 1, pp. 294-296.

\section{Article Google Scholar}

\section{Author information}

Affiliations

1. Khristianovich Institute of Theoretical and Applied

Mechanics, Siberian Branch, Russian Academy of Sciences, Novosibirsk, 630090, Russia

V. I. Kuzmin \& D. V. Sergachev

2. Branch of the Belarusian National Technical University, Institute of Improvement of Professional Skills and Staff Retraining on New Directions of Engineering, Minsk, 220014, Belarus

N. A. Rudenskaya

3. Polotsk State University, Polotsk, 211440, Belarus

N. V. Sokolova

Corresponding author

Correspondence to $\underline{\text { N. A. Rudenskaya. }}$ 


\section{Additional information}

Original Russian Text (C) V.I. Kuzmin, N.A. Rudenskaya, N.V. Sokolova, D.V. Sergachev, 2017, published in Trenie i Iznos, 2017, Vol. 38, No. 5, pp. 467474 .

\section{About this article}

Cite this article

Kuzmin, V.I., Rudenskaya, N.A., Sokolova, N.V. et al. Wear resistance coatings of nickel alloys obtained by supersonic plasma torch. J. Frict. Wear 38, 395400 (2017). https://doi.org/10.3103/S1068366617050063

- Received05 January 2017

- Published21 October 2017

- Issue DateSeptember 2017

- DOI https://doi.org/10.3103/S1068366617050063

Keywords

- plasma torch

- atmospheric plasma spraying

- coating

- resistance to wear and tear

- abrasive friction

- powder ring injector

- gas dynamic focusing

- powder material 